\title{
AN EQUATION ALTERNATELY OF RETARDED AND ADVANCED TYPE
}

\author{
KENNETH L. COOKE AND JOSEPH WIENER
}

\begin{abstract}
We study a differential equation with the argument $2[(t+1) / 2]$, where $[\cdot]$ denotes the greatest-integer function. The argument deviation $\tau(t)=t-$ $2[(t+1) / 2]$ is a function of period 2 and equals $t$ for $-1 \leqslant t<1$. It changes its sign in each interval $2 n-1 \leqslant t<2 n+1$.
\end{abstract}

1. Introduction. This note continues the investigation of differential equations with piecewise constant arguments (EPCA) originated by K. L. Cooke and J. Wiener [3], and S. M. Shah and J. Wiener [4]. They are closely related to impulse and loaded equations and, especially, to difference equations of a discrete argument. These equations have the structure of continuous dynamical systems within intervals of certain length. Continuity of a solution at a point joining any two consecutive intervals then implies recursion relations for the solution at such points. The equations are thus similar in structure to those found in certain "sequential-continuous" models of disease dynamics as treated by S. Busenberg and K. L. Cooke [2]. The above works show that all types of EPCA share similar characteristics. First of all, it is natural to pose the initial-value problem for such equations not on an interval but a number of individual points. Secondly, two-sided solutions exist for all types of EPCA. Finally, since EPCA combine the features of both differential and difference equations, their asymptotic behavior as $t \rightarrow \infty$ resembles in some cases the growth of solutions of differential equations, while in others it inherits the properties of difference equations.

2. Main results. We consider the equation

$$
x^{\prime}(t)=a x(t)+a_{0} x(2[(t+1) / 2]), \quad x(0)=c_{0},
$$

where $[\cdot]$ is the greatest-integer function. The argument deviation

$$
\tau(t)=t-2[(t+1) / 2]
$$

is negative for $2 n-1 \leqslant t<2 n$, and positive for $2 n<t<2 n+1$ ( $n$ is an integer).

Received by the editors February 10, 1986. Some of the contents of this article were presented at the International Congress of Mathematicians, Berkeley, California, August, 1986.

1980 Mathematics Subject Classification (1985 Revision). Primary 34K05, 34K15; Secondary 34K20.

The first author was partially supported by NSF Grant DMS-8405383.

The second author was partially supported by U. S. Army Research Grant DAAG29-84-G-0034. 
Therefore, "equation (1)" is of considerable interest: on each interval [ $2 n-1,2 n+$ 1 ) it is of alternately advanced and retarded type. "Equation (1)" is of advanced type on $[2 n-1,2 n)$ and of retarded type on $(2 n, 2 n+1)$.

DEFINITION. A solution of "equation (1)" on $[0, \infty)$ is a function $x(t)$ that satisfies the conditions:

(i) $x(t)$ is continuous on $[0, \infty)$.

(ii) The derivative $x^{\prime}(t)$ exists at each point $t \in[0, \infty)$, with the possible exception of the points $t=2 n-1(n=1,2, \ldots)$, where one-sided derivatives exist.

(iii) "Equation (1)" is satisfied on each interval $2 n-1 \leqslant t<2 n+1$.

In this paper, we show that problem (1) has a unique solution on $[0, \infty)$ and a unique backward solution on $(-\infty, 0]$. Also, we determine the set of $\left(a, a_{0}\right)$ for which the zero solution is asymptotically stable as $t \rightarrow+\infty$, and the set of $\left(a, a_{0}\right)$ such that all nontrivial solutions have no zeros in $(-\infty, \infty)$. The set of bounded solutions is characterized. Furthermore, the same equation with variable coefficients $a(t)$, $a_{0}(t)$ is examined, the condition for existence of a unique solution on $[0, \infty)$ is determined, and conditions are found under which all solutions are oscillatory.

Let

$$
\lambda(t)=e^{a t}+a^{-1} a_{0}\left(e^{a t}-1\right), \quad \lambda_{1}=\lambda(1), \quad \lambda_{-1}=\lambda(-1) .
$$

TheOREM 1. Problem (1) has on $[0, \infty)$ a unique solution

$$
x(t)=\lambda(\tau(t))\left(\lambda_{1} / \lambda_{-1}\right)^{[(t+1) / 2]} c_{0}, \quad \text { if } \lambda_{-1} \neq 0,
$$

where $\tau(t)$ is given by (2).

Proof. Assuming that $x_{n}(t)$ is a solution of "equation (1)" on the interval $2 n-1 \leqslant t<2 n+1$, with the condition $x_{n}(2 n)=c_{2 n}$, we have

$$
x_{n}^{\prime}(t)=a x_{n}(t)+a_{0} c_{2 n} .
$$

The general solution of this equation on the given interval is

$$
x_{n}(t)=e^{a(t-2 n)} c-a^{-1} a_{0} c_{2 n},
$$

with an arbitrary constant $c$. Putting here $t=2 n$ gives $c_{2 n}=c-a^{-1} a_{0} c_{2 n}$ and

$$
x_{n}(t)=\lambda(t-2 n) c_{2 n} \text {. }
$$

For $t=2 n-1$, we have

$$
x_{n}(2 n-1)=c_{2 n-1}=\lambda_{-1} c_{2 n}, \quad c_{2 n}=\lambda_{-1}^{-1} c_{2 n-1},
$$

and for $t=2 n+1$,

$$
x_{n}(2 n+1)=c_{2 n+1}=\lambda_{1} c_{2 n} .
$$

Hence,

$$
\begin{aligned}
& c_{2 n+1}=\left(\lambda_{1} / \lambda_{-1}\right) c_{2 n-1}=\left(\lambda_{1} / \lambda_{-1}\right)^{n} c_{1}, \\
& c_{2 n}=\lambda_{-1}^{-1} c_{2 n-1}=\lambda_{-1}^{-1}\left(\lambda_{1} / \lambda_{-1}\right)^{n-1} c_{1} .
\end{aligned}
$$


From (5) it follows that $x_{0}(t)=\lambda(t) c_{0}$ and $x_{0}(1)=c_{1}=\lambda_{1} c_{0}$ for $0 \leqslant t<1$. Therefore, $c_{2 n}=\left(\lambda_{1} / \lambda_{-1}\right)^{n} c_{0}$ and

$$
x_{n}(t)=\lambda(t-2 n)\left(\lambda_{1} / \lambda_{-1}\right)^{n} c_{0},
$$

where $\lambda(t), \lambda_{1}$, and $\lambda_{-1}$ are given by (3). Formula (6) is equivalent to (4). It was obtained with the implicit assumption $a \neq 0$, but the limiting case of (4) as $a \rightarrow 0$ is the solution of problem (1) with $a=0$, if $a_{0} \neq 1$.

THEOREM 2. The solution of problem (1) has a unique backward continuation on $(-\infty, 0]$ given by formula (4) if $\lambda_{1} \neq 0$.

Proof. If $x_{-n}(t)$ denotes the solution of (1) on $-2 n-1 \leqslant t<-2 n+1$ satisfying the condition $x_{-n}(-2 n)=c_{-2 n}$, then from the equation $x_{-n}^{\prime}(t)=a x_{-n}(t)+a_{0} c_{-2 n}$ it follows that

$$
x_{-n}(t)=e^{a(t+2 n)} c-a^{-1} a_{0} c_{-2 n} .
$$

At $t=-2 n$ we get

$$
c=\left(1+a^{-1} a_{0}\right) c_{-2 n}, \quad x_{-n}(t)=\lambda(t+2 n) c_{-2 n} .
$$

For $t=-2 n+1$,

$$
x_{-n}(-2 n+1)=c_{-2 n+1}=\lambda_{1} c_{-2 n}, \quad c_{-2 n}=\lambda_{-1}^{-1} c_{-2 n+1} .
$$

For $t=-2 n-1$,

$$
x_{-n}(-2 n-1)=c_{-2 n-1}=\lambda_{-1} c_{-2 n} \text {. }
$$

Hence,

$$
c_{-2 n-1}=\left(\lambda_{-1} / \lambda_{1}\right) c_{-2 n+1}=\left(\lambda_{-1} / \lambda_{1}\right)^{n} c_{-1}
$$

and

$$
c_{-2 n}=\lambda_{-1}^{-1}\left(\lambda_{-1} / \lambda_{1}\right)^{n-1} c_{-1}
$$

Finally, on $-1 \leqslant t<0$ we have

$$
x_{0}(t)=\lambda(t) c_{0}, \quad x_{0}(-1)=c_{-1}=\lambda_{-1} c_{0} .
$$

Thus, $c_{-2 n}=\left(\lambda_{-1} / \lambda_{1}\right)^{n} c_{0}$ and

$$
x_{-n}(t)=\lambda(t+2 n)\left(\lambda_{-1} / \lambda_{1}\right)^{n} c_{0} .
$$

For $t \leqslant 0$, this formula coincides with.(4).

THEOREM 3. The solution $x=0$ of "equation (1)" is asymptotically stable as $t \rightarrow+\infty$ if and only if $\left|\lambda_{1} / \lambda_{-1}\right|<1$.

Proof. Since $|\tau(t)| \leqslant 1$ and $\lambda(\tau)$ is continuous, the function $\lambda(\tau(t))$ is bounded for all $t$. The proof then follows easily from (4). 
THEOREM 4. The solution $x=0$ of "equation (1)" is asymptotically stable as $t \rightarrow+\infty$ if and only if any one of the following hypotheses is satisfied:

$$
\begin{aligned}
& a<0, \quad a_{0}>-\frac{a\left(e^{2 a}+1\right)}{\left(e^{a}-1\right)^{2}} \text { or } a_{0}<-a ; \\
& a>0, \quad-\frac{a\left(e^{2 a}+1\right)}{\left(e^{a}-1\right)^{2}}<a_{0}<-a ; \\
& a=0, \quad a_{0}<0 .
\end{aligned}
$$

Proof. For the function $\lambda(t)$ we have $\lambda^{\prime}(t)=\left(a+a_{0}\right) e^{a t}$. If $a+a_{0}>0$, then $\lambda(t)$ is increasing, and assuming $\lambda(-1)>0$ leads to $\lambda(1)>\lambda(-1)$, that is, $\lambda_{1} / \lambda_{-1}$ $>1$. The conditions $a+a_{0}>0$ and $\lambda(-1)>0$ can be written as $-a<a_{0}<$ $a /\left(e^{a}-1\right)$. In this case, the solution $x=0$ is unstable. The case $a+a_{0}<0$, $\lambda(-1)<0$ is impossible. Indeed, the inequalities $a_{0}<-a$ and $a_{0}>a /\left(e^{a}-1\right)$ are inconsistent because $-a<a /\left(e^{a}-1\right)$. From $a+a_{0}>0$ and $\lambda(-1)<0$ it follows that

$$
a_{0}>a /\left(e^{a}-1\right)
$$

The inequality $\lambda_{1} / \lambda_{-1}<1$ implies

$$
e^{a}+\frac{a_{0}}{a}\left(e^{a}-1\right)>e^{-a}+\frac{a_{0}}{a}\left(e^{-a}-1\right)
$$

which is equivalent to $a+a_{0}>0$. On the other hand, $\lambda_{1} / \lambda_{-1}>-1$ gives

$$
e^{a}+\frac{a_{0}}{a}\left(e^{a}-1\right)<-e^{-a}-\frac{a_{0}}{a}\left(e^{-a}-1\right),
$$

whence $\left(e^{2 a}+1\right) /\left(e^{a}-1\right)^{2}<-a_{0} / a$. If $a>0$, then

$$
a_{0}<-\frac{a\left(e^{2 a}+1\right)}{\left(e^{a}-1\right)^{2}}
$$

This contradicts (7). For $a<0$, we have

$$
a_{0}>-\frac{a\left(e^{2 a}+1\right)}{\left(e^{a}-1\right)^{2}}
$$

and since $a /\left(e^{a}-1\right)<-a\left(e^{2 a}+1\right) /\left(e^{a}-1\right)^{2}$, hypothesis (i) ensures asymptotic stability of $x=0$. Finally, the conditions $a+a_{0}<0$ and $\lambda(-1)>0$ simply reduce to $a_{0}<-a$. The same result follows from the inequality $\lambda_{1}<\lambda_{-1}$. Furthermore, from $\lambda_{1}>-\lambda_{-1}$ we obtain

$$
-\frac{e^{2 a}+1}{\left(e^{a}-1\right)^{2}}<\frac{a_{0}}{a} .
$$

For $a>0$, this confirms hypothesis (ii). The case $a<0$ again leads to $a_{0}<-a$. If $a=0$, then $\left|\lambda_{1} / \lambda_{-1}\right|=\left|\left(1+a_{0}\right) /\left(1-a_{0}\right)\right|<1$ holds for $a_{0}<0$.

THEOREM 5. All nontrivial solutions of "equation (1)" have no zeros in $(-\infty, \infty)$ if and only if

$$
-\frac{a e^{a}}{e^{a}-1}<a_{0}<\frac{a}{e^{a}-1}
$$


Proof. The conclusion follows from the fact that $\lambda(t)$ is a monotone function if $a+a_{0} \neq 0$; then solution (4) has no zeros if and only if $\lambda(-1)$ and $\lambda(1)$ are of the same sign, that is, $\lambda_{1} / \lambda_{-1}>0$. The case $\lambda_{-1}<0, \lambda_{1}<0$ is impossible since it leads to inconsistent inequalities

$$
a_{0}<-\frac{a e^{a}}{e^{a}-1}, \quad a_{0}>\frac{a}{e^{a}-1} .
$$

On the contrary, the case $\lambda_{-1}>0, \lambda_{1}>0$ yields (8). If $a+a_{0}=0$, the only solution of problem (1) is $x(t)=c_{0}$.

THEOREM 6. The problem

$$
x^{\prime}(t)=a(t) x(t)+a_{0}(t) x(2[(t+1) / 2]), \quad x(0)=c_{0}
$$

has a unique solution on $[0, \infty)$ if $a(t)$ and $a_{0}(t)$ are continuous for $t \geqslant 0$, and

$$
\int_{2 n-1}^{2 n} u^{-1}(t) a_{0}(t) d t \neq u^{-1}(2 n), \quad n=1,2, \ldots,
$$

where $u^{-1}$ is the reciprocal of $u$ and $u(t)=\exp \left(\int_{0}^{t} a(s) d s\right)$.

THEOREM 7. The functional differential inequality

$$
x^{\prime}(t)+p(t) x(t)+q(t) x(2[(t+1) / 2]) \leqslant 0
$$

with $p(t)$ and $q(t)$ continuous on $[0, \infty)$ has no eventually positive solution if

$$
\lim _{n \rightarrow \infty} \sup \int_{2 n}^{2 n+1} q(t) \exp \left(\int_{2 n}^{t} p(s) d s\right) d t>1 .
$$

Proof. Following [1], we prove that the existence of an eventually positive solution leads to a contradiction. To this end suppose that $x(t)$ is a solution of (10) such that $x(t)>0$ for $t \geqslant 2 n$, where $n$ is a sufficiently large integer. For $2 n-1 \leqslant t$ $<2 n+1$, inequality (10) becomes

$$
x^{\prime}(t)+p(t) x(t)+q(t) x(2 n) \leqslant 0,
$$

or

$$
y^{\prime}(t)+q(t) \exp \left(\int_{2 n}^{t} p(s) d s\right) y(2 n) \leqslant 0
$$

where $y(t)=x(t) \exp \left(\int_{2 n}^{t} p(s) d s\right)$. Integrating (12) from $2 n$ to $2 n+1$, we have

$$
y(2 n+1) \leqslant y(2 n)\left(1-\int_{2 n}^{2 n+1} q(t) \exp \left(\int_{2 n}^{t} p(s) d s\right) d t\right) .
$$

Since $y(t)>0$ for $t \geqslant 2 n$, then

$$
1-\int_{2 n}^{2 n+1} q(t) \exp \left(\int_{2 n}^{t} p(s) d s\right) d t>0
$$

or

$$
\lim _{n \rightarrow \infty} \sup \int_{2 n}^{2 n+1} q(t) \exp \left(\int_{2 n}^{t} p(s) d s\right) d t \leqslant 1 .
$$

This contradicts (11). So, (10) has no eventually positive solution. 
THEOREM 8. If condition (11) is satisfied, the functional differential inequality

$$
x^{\prime}(t)+p(t) x(t)+q(t) x(2[(t+1) / 2]) \geqslant 0
$$

has no eventually negative solution.

From Theorems 7 and 8 it follows that subject to hypothesis (11), the equation

$$
x^{\prime}(t)+p(t) x(t)+q(t) x(2[(t+1) / 2])=0
$$

has no eventually positive or eventually negative solutions and therefore we are led to the following conclusion.

THEOREM 9. Subject to condition (11), "equation (14)" has oscillatory solutions only.

COROllary. “Equation (9)" has only oscillatory solutions on $[0, \infty)$ if

$$
\lim _{n \rightarrow \infty} \inf \int_{2 n}^{2 n+1} a_{0}(t) \exp \left(-\int_{2 n}^{t} a(s) d s\right) d t<-1
$$

REMARK. Condition (15) is sharp. For "equation (1)" with constant coefficients, (15) becomes $a_{0}<-a e^{a} /\left(e^{a}-1\right)$ which is, according to (8), one of the two "best possible" conditions for oscillation.

THEOREM 10. Inequality (10) has no eventually negative solution if

$$
\lim _{n \rightarrow \infty} \inf \int_{2 n-1}^{2 n} q(t) \exp \left(\int_{2 n}^{t} p(s) d s\right) d t<-1 .
$$

Proof. Suppose that $x(t)$ is a solution of (10) such that $x(t)<0$ for $t \geqslant 2 n-1$, where $n$ is a sufficiently large integer. Integrating (12) from $2 n-1$ to $2 n$ gives

$$
y(2 n)\left(1+\int_{2 n-1}^{2 n} q(t) \exp \left(\int_{2 n}^{t} p(s) d s\right) d t\right) \leqslant y(2 n-1),
$$

and since $y(t)<0$ for $t \geqslant 2 n-1$, then

$$
1+\int_{2 n-1}^{2 n} q(t) \exp \left(\int_{2 n}^{t} p(s) d s\right) d t>0
$$

or

$$
\lim _{n \rightarrow \infty} \inf \int_{2 n-1}^{2 n} q(t) \exp \left(\int_{2 n}^{t} p(s) d s\right) d t \geqslant-1,
$$

which contradicts (16).

THEOREM 11. If condition (16) is satisfied, (13) has no eventually positive solution.

TheOREM 12. Subject to condition (16), "equation (14)" has oscillatory solutions only.

COROllaRY. "Equation (9)" has only oscillatory solutions on $[0, \infty)$ if

$$
\lim _{n \rightarrow \infty} \sup \int_{2 n-1}^{2 n} a_{0}(t) \exp \left(-\int_{2 n}^{t} a(s) d s\right) d t>1 \text {. }
$$

REMARK. Condition (17) is sharp. For "equation (1)" with constant coefficients, (17) becomes $a_{0}>a /\left(e^{a}-1\right)$ which is, according to (8), one of the two "best possible" conditions for oscillation. 
THEOREM 13. If $a_{0}>a /\left(e^{a}-1\right)$, then solution (4) with the condition $x(0)=c_{0}$ has precisely one zero in each interval $2 n-1<t<2 n$ with integral endpoints. If $a_{0}<-a e^{a} /\left(e^{a}-1\right)$, then (4) has precisely one zero in each interval $2 n<t<2 n+1$.

THEOREM 14. All solutions of "equation (1)" that are bounded on $-\infty<t<\infty$ and that do not tend to zero as $t \rightarrow \pm \infty$ are periodic. They exist only for $a_{0}=-a$ or $a_{0}=-a\left(e^{2 a}+1\right) /\left(e^{a}-1\right)^{2}$. In the first case, the solutions are constant; and in the second case, they are of period 4.

Proof. The conclusions follow from (4) and from the condition $\left|\lambda_{1} / \lambda_{-1}\right|=1$ which is necessary and sufficient for $x(t)$ to be bounded and not vanish.

\section{REFERENCES}

1. A. R. Aftabizadeh and J. Wiener, Oscillatory properties of first order linear functional differential equations, Applicable Anal. 20 (1985), 165-187.

2. S. Busenberg and K. L. Cooke, Models of vertically transmitted diseases with sequential-continuous dynamics, Nonlinear Phenomena in Mathematical Sciences (V. Lakshmikantham, Ed.), Academic Press, New York, 1982, pp. 179-187.

3. K. L. Cooke and J. Wiener, Retarded differential equations with piecewise constant delays, J. Math. Anal. Appl. 99 (1984), 265-297.

4. S. M. Shah and J. Wiener, Advanced differential equations with piecewise constant argument deviations, Internat. J. Math. Math. Sci. 6 (1983), 671-703.

Department of Mathematics, Pomona College, Claremont, California 91711

Department of Mathematics, Pan American University, Edinburg, TeXas 78539 